\title{
Conditional Independence in Categories (Abstract)
}

\author{
Alex Simpson \\ LFCS, School of Informatics, \\ University of Edinburgh, \\ Scotland, UK. \\ Alex.Simpson@ed.ac.uk
}

In this talk I shall discuss a general category-theoretic structure for modelling conditional independence. The standard notion of conditional independence in probability theory provides a motivating example. But other rather different examples arise in many contexts: computability theory, nominal sets (used to model 'names' in computer science), separation logic (used to reason about heap memory in computer science), and others.

Category-theoretic structure common to these examples can be axiomatized by the notion of a category with local independent products, which combines fibrational and symmetric monoidal structure in a somewhat particular way. In the talk I shall expound this notion, and I shall present several illustrative examples of such structure. If time permits, I may also describe some curious connections with topos theory. 